\title{
SYNCHRONOUS MALIGNANT TUMORS OF THE COLON
}

\author{
Deenichin G., R. Dimov, V. Molov, Ch. Stefanov \\ Department of Surgery III Medical University, Plovdiv
}

Reviewed by: Assoc. Prof. R. Madjov, MD, PhD

\begin{abstract}
According to literature the synchronous malignant tumors of the colon are $2-11 \%$ from all cases of sporadic nonhereditary colorectal cancer. This big difference in the frequency is due to variations in the accuracy of the used diagnostic methods, and also to the intentional search for associated lesions. The synchronous tumors have different biology and prognosis than solitary tumors. Investigations performed with the methods of genetic engineering over p53 gene abnormalities strongly suggest that the great majority of synchronous colonic adenocarcinomas arise as independent neoplasms and their worsened prognosis is not a result of unusually early metastatic spread. The aim of our one-year retrospective study was to find out the frequency of these tumors towards all patients with colorectal cancer, the time for correct diagnosis (intraoperationem or before the operation), and the peculiarities in the surgical tactics. We analyzed retrospectively 106 patients with colorectal cancer treated in the Department of Surgery III Medical University Plovdiv for one year period (01.06.2006-01.06.2007), 61 men (57.5\%) with mean age 64+-2.5 years and $45(42.5 \%)$ women with mean age 61+-3.1 year.
\end{abstract}

Keywords: Synchronous malignant tumors, Colon

From the whole number of 106 patients with cancer of the colon and rectum, 6 were with synchronous malignant tumors of the colon $(5.66 \%)$. Four of these six cases were with cancer of the sigmoid colon and descendent colon, and two with sigmoid colon and ascendent colon localization. In three of the patients the diagnosis of multiple pathology was confirmed preoperatively, and in the other three-intraoperationem because of the impossibility to perform full colonoscopy due to the almost total obstruction of the distal tumor. The operation in the group of patients with tumors of the sigmoid and the descendent colon was left hemicolectomy, while in the other group with combined tumors of the sigmoid and ascendent colon -right hemicolectomy and resection of the sigmoid colon. All six patients operated for synchronous tumors of the colon were in stage III (Dukes $\mathrm{C}$ colon cancer).

The frequency of the synchronous tumors of the colon is high-according to our data is $5.66 \%$ from all cancers of the colon, and because of the bettered diagnosis and the bigger absolute numbers of the tumors of the colorectal zone is with a tendency to grow. The genetic engineering proved the multicentricity of the process, and the hystoanalisis show, that the patients with colorectal cancer have an unstable epithelium and an uncommon predisposition to develop several mucosal alterations synchronously or metachronously. We emphasize the need for a full evaluation of the colon in all patients with colorectal carcinoma. In the case of incomplete preoperative evaluation, intraoperative colonoscopy is to be considered; if this is not feasible it should be performed one month after surgery.
We consider the total colectomy to be indicated in specific situations, and not as an obligatory element in the process of treatment of the synchronous malignant tumors of the colon. It is necessary to think about the possibility for such kind of pathology and to seek it hard.

\section{INTRODUCTION}

The synchronous malignant tumors of the colon are unusual, but well known phenomenon and according to literature data are $2-11 \%$ from all cases of sporadic nonhereditary colorectal cancer (1), 1.5-7.6\% (2), 1.7-9.3\% (3), $5-10 \%$ (4). This big difference in the frequency is due to variations in the accuracy of the used diagnostic methods, and also the intentional search for associated lesions. The unsuccess of the correct diagnostics of the synchronous tumors results in mistakes in the treatment tactics and worsens the prognosis (5). There is an opinion, based on investigation of 160 patients with total amount of 339 synchronous tumors, according to which the patients with solitary tumors of the colon and rectum have similar development of the disease and survival rate as those with synchronous tumors (6). Much more accepted is the opinion, that the synchronous tumors have different biology and prognosis than solitary ones. Investigations performed with the methods of genetic engineering over p53 gene abnormalities using the polymerase chain reaction, followed by analysis confirm the polymorphism and strongly suggest that the great majority of synchronous colonic adenocarcinomas 
arise as independent neoplasma and their worsened prognosis is not a result of unusually early metastasis spread (1). For clinical purposes in practice for staging of the colorectal cancer in the abdominal surgery is used successfully the three grade scale of Dukes, including A, B and C, later modified by Astler-Coller, they include one more grade D. Nowadays is used the latest modification of the scale from 1978y., proposed from Gunderson and Sosin with subgroups B1 and B2 in stage Duke B, and C1 and C2 in stage Dukes C.

Modified Duke's scale:

1. Modified Duke A colon cancer-the tumor penetrates only the mucosal membrane of the intestinal wall.

2. Modified Duke B colon cancer:

B1-the tumor penetrates in, but not through muscularis propria of the intestinal wall.

B2-the tumor penetrates in and through the muscularis propria of the intestinal wall.

3. Modified Duke $\mathrm{C}$ colon cancer:

C1-the tumor penetrates in, but not through the muscularis propria; there is evidence for tumor changes in the lymph nodes.

C2-the tumor penetrates in and through the muscularis propria of the intestinal wall; there is evidence for tumor changes in the lymph nodes.

4. Modified Duke D colon cancer-the tumor is widespread far away of the lymph nodes borders to the other organs-liver, lung, bones etc. The tumor can be in any size including or not including changed lymph nodes.

For diagnostic purposes there are proposed different approaches -roentgen images with contract material, fibro colonoscopy and thorough exploration during the operation. According to some big studies on the subject the investigation with barium enema give unsatisfactory results, the false negative results from fibro colonoscopy are about $30 \%$, while the intraoperative palpation finds out nearly $60 \%$ of the unexpected synchronous tumors (7). It is considered, that the fibro colonoscopy combined with a thorough intraoperative palpation of the whole colon are crucial for the early diagnosis of the synchronous colorectal cancer (8).

\section{AIM}

The aim of our one-year retrospective study was to find out the frequency of these tumors towards all patients with colorectal cancer, the time and the method for confirming the correct diagnosis (intraoperationem or before the operation), and the peculiarities of the surgical tactics.

\section{MATERIALS AND METHODS}

We analyzed retrospectively 106 patients with colorectal cancer treated in the Department of Surgery III Medical University Plovdiv for one year period
(01.06.2006-01.06.2007), 61 men (57.5\%) with mean age $64+-2.5$ years and $45(42.5 \%)$ women with mean age $61+-3.1$ year.

\section{RESULTS AND DISCUSSION}

From the whole number of 106 patients operated in the Department for one-year period for colorectal cancers, 6 were with synchronous malignant tumors of the colon (5.66\%). Four of these six cases were with cancer of the sigmoid colon and descendent colon, and two with sigmoid colon and ascendant colon localization. In three of the patients the diagnosis of multiple pathology was confirmed preoperatively, and in the other three-intraoperationem because of the impossibility to perform full colonoscopy due to the almost total obstruction of the distal tumor. The operation in the group of patients with tumors of the sigmoid and descendent colon was left hemicolectomy, while in the group with combined cancer of the sigmoid colon and ascendant colon-right hemicolectomy and resection of the sigmoid colon. All six patients operated for synchronous tumors of the colon were in stage III (Duke's C colon cancer).

\section{CONCLUSIONS}

The frequency of the synchronous tumors of the colon is high-according to our data is $5.66 \%$ from all cancers of the colon, and because of the bettered diagnosis and the higher absolute number of tumors of the colorectal zone is with a tendency to grow. The genetic engineering proved the multicentricity of the process, and the hystoanalisis show, that the patients with colorectal cancer have an unstable epithelium and an uncommon predisposition to develop several mucosal alterations synchronously or metachronously (9). We emphasize the need for a full evaluation of the colon in all patients with colorectal cancer. In cases of incomplete preoperative evaluation, intraoperative colonoscopy is to be considered; if this not feasible it should be performed one month after surgery. We consider the total colectomy to be indicated in specific situations, and not as an obligatory element in the process of treatment of the synchronous malignant tumors of the colon. It is necessary to think about the possibility for such kind of pathology and to seek it hard.

\section{REFERENCES}

1. Koness RJ, King TC, Scheechter S, McLean SF, Lodowsky C and HJ Wanebo. Synchronous colon carcinomas: molecular-genetic evidence for milticentricity. Ann Surg Oncology 1066; 3(2): 136-143.

2. Fegis G, Ramacciato G, Indinnimeo M, De Angelis R and P Barillari. Synchronous large bowel cancer: a series of 47 cases. Ital J Surg Sci 1989; 19(1):23-8. 
3. Schaal JC, Mondino JC, Paris F, Piat JM and D Jaeck. Synchronous colorectal cancers. J Chir (Paris) 1991 Nov; 128(11):476-80.

4. Pedroni M, Tamassia MG, Percesepe A, Roncucci L, Benatti P, Lanza G Jr, Gafa R et al. Human cancer. Microsatellite instability in multiple colorectal tumors. Int J Cancer 1999 Nov; 81(1): $1-5$.

5. Feffer VF, Sprekelsen BJC, Diana FCA, Perez AJ, Prado VA, Coret GMJ, Puchades GF and PR Trullenque. Multiple colon tumors. Diagnosis and follow-up of 450 patients with colorectal carcinoma. Rev Esp Enferm Dig 1997 Oct; 89(10):759-63.

6. Passman MA, Pommier RF and JT Vetto. Synchronous colon primaries have the same prognosis as solitary colon cancers. Dis Colon Rectum 1996 Mar; 39(3):329-334.

7. Chen HS and Sheen-Chen SM. Synchronous and "early" metachronous colorectal adenocarcinoma: analysis of prognosis and current trends. Dis Colon Rectum 2000 Aug;43(8):1093-9.

8. Tuscano D, D'Amore L, Negro P, Scaccia M, Talacito C, Gosseti F, Flati D and M Carboni. Double synchronous occluding tumors of the large bowel: A report of three cases. Surgery Today 1996 Nov; 26(11):926-8.

9. Martines GEE, Pena REJP, Villanueva-Saenz E, Alvarez-Tostado FGF and M Arenas-Sanchez. Synchronous neoplasmas in colorectal cancer. Rev Gastroenterol Mex. 2000; 65(2):63-8. 